\title{
Comment
}

Neuro epidemiology

Neuroepidemiology 2014;43:26-27

DOI: $10.1159 / 000366428$

\section{MMSE 2.0: A New Approach to an Old Measure}

\section{Steven M. Albert}

Department of Behavioral and Community Health Sciences, Graduate School of Public Health, University of Pittsburgh, Pittsburgh, Pa., USA

The Mini-Mental State Examination (MMSE) is the industry standard for brief cognitive assessments of older adults. It is meant to be 'a practical method for grading the cognitive state of patients for the clinician', as the title of its original paper states [1], but it is commonly used in epidemiologic cohorts to identify incident dementia and cognitive decline. Its 11 questions assess 5 cognitive domains: orientation, registration, attention and calculation, recall, and language. Scores range from 0 to 30, with a score of 23 or lower indicating possible cognitive impairment and the need for further assessment. The measure has been used in thousands of published studies. It has authorized translations in over 70 languages. It has been shortened and expanded [2], normed for different age, education, and race groups [3], and subjected to extensive psychometric investigation and validation efforts [4].

Regression-based T-scores for MMSE performance have been developed to help mitigate confounding associated with literacy and education, a challenge for all neuropsychological assessment. Yet these kinds of adjustments only go so far. They do not help with floor and ceiling effects or the differential sensitivity to cognitive change at the upper and lower ranges of MMSE scores. Variation in sensitivity to change is seen in the different clinical meaning of a 1-point MMSE change depending on a person's initial score. With an initial high score of 27-30, a 1-point decline may not be clinically meaningful; however, a 1-point decline in people scoring in the intermediate range of scores (10-20) can indicate substantial declines in cognitive ability. These 'metrological' or psychometric properties compromise the value of the measure for epidemiologic study and can lead to false inferences about cognitive change without sophisticated statistical modeling.

Philipps et al. [5] have addressed this problem with a normalizing transformation of MMSE scores. They estimated these scores and validated them using two population-based studies (the PAQUID and the Three-City Study in southwestern France) with a 20 -year follow-up to estimate cognitive change. The cohorts are heterogeneous and include normal and pathological aging as well as samples including only participants meeting criteria for dementia.

\section{KARGER}

E-Mail karger@karger.com

www.karger.com/ned
The normalizing transformation corrects for the curvilinearity of MMSE performance relative to true change in underlying cognitive status. The normalizing transformation, scaled from 0 to 100 , converts crude scores of 20,24, and 28, for example, to $37.37,51.44$, and 74.61, respectively, showing that a 4-point difference in crude MMSE scores represents an actual difference of roughly 14 points between MMSE scores of 20 and 24 and more than 23 points between MMSE scores of 24 and 28.

This approach to the MMSE is likely to have great value. The normalizing transformation, conveniently presented as a look-up table in figure 3 of their paper, 'makes possible the use of standard statistical methods ... to study MMSE scores as a dependent variable.' 'By changing the interval between two successive values, we counteract the metrological problems with MMSE and provide a score that uses the same information as MMSE but with corrected intervals between successive scores.'

The value of the transformation can be seen in the effect of education on estimates of cognitive decline. Phillips's simulations show that raw score analyses underestimate the effect of educational level on MMSE performance at baseline but overestimate the effect of education on change, 'leading to incorrect conclusions regarding EL [educational level] as a risk factor of cognitive decline'.

This last point should not go unnoticed by neuroepidemiologists. A great deal of research on 'cognitive reserve' and social determinants of cognitive status in old age has demonstrated the key role of educational attainment early in life for risk of dementia and trajectories of cognitive decline with age. The role of education, occupation, and cognitive engagement for cognitive outcomes is a widely reported finding and not limited to the MMSE as a cognitive outcome. Could the effect of education on dementia risk actually be partly an artifact of the measurement properties of the MMSE? The normalizing transformation proposed by Phillips et al. [5] suggests at a minimum that studies using raw MMSE scores as outcomes should be reexamined and reestimated using this MMSE normalizing transformation.

Another important use of the normalizing transformation is a more accurate comparison of risk factor effects across different longitudinal studies. Phillips et al. [5] suggest that this transformation of MMSE scores should apply to any valid translation of the MMSE, even though it was developed and validated on the Frenchlanguage version. They provide both a convenient look-up table as well as an R package that will compute the normalized transformation for the crude MMSE scale.

In short, this normalizing transformation of the MMSE should help improve the accuracy of studies of cognitive change in old age. A first charge for investigators is to determine if their model predictions change if they use these normalized scores as outcomes rather than raw scores. A second charge is to reexamine education 
to determine how much declines in cognition in old age can be truly attributed to differences in education over the life span, and how much may be artifacts of the curvilinear properties of cognitive assessments such as the MMSE.

\section{References}

1 Folstein MF, Folstein SE, McHugh PR: 'Mini-mental state': a practical method for grading the cognitive state of patients for the clinician. J Psychiatr Res 1975;12:189-198.
2 Folstein MF, Folstein SE, Fanjiang G: Mini-Mental State Examination: Clinical Guide. Lutz, Psychological Assessment Resources, 2001.

- 3 Crum RM, Anthony JC, Bassett SS, Folstein MF: Population-based norms for the Mini-Mental State Examination by age and educational level. J Am Med Assoc 1993;269:2386-2391.

4 Tombaugh TN, McIntyre NJ: The Mini-Mental State Examination: a comprehensive review. J Am Geriatr Soc 1992;40:922-935.

-5 Philipps V, Amieva H, Andrieu S, Dufouil C, Berr C, Dartigues JF, Jacqmin-Gadda H, Proust-Lima C: Normalized Mini-Mental State Examination for assessing cognitive change in population-based brain aging studies. Neuroepidemiology 2014;43:15-25. 